Helgoländer wiss. Meeresunters. 17, 94-107 (1968)

\title{
Strömungsmessungen in der Deutschen Bucht bei Sturmfluten
}

\author{
Hans Gienapp und Gerhard Tomczak \\ Deutsches Hydrographisches Institut, Hamburg
}

\begin{abstract}
Current measurements in the German Bight during storm surges. During the storm surges of the winter period 1965/66 current measurements were conducted in eight geographical positions at two, sometimes also three, different depth layers. The evaluation of the measured data yielded current fields of positive and negative storm surges (at westerly or easterly winds, respectively) and spectra of the internal motion of the German Bight in the oscillation period from 3 to 90 hours. Typical storm surge conditions with strong south-west and north-west winds prevailed in the period from 29 October to 4 November, 1965. Current meters were then placed in the following positions: Vortrapp-Tief, Außeneider, P 8, Helgoland, Elbe 1, Weser light vessel, Norderney, Borkumriff. Their recordings indicate that the transport of water into the areas off the mouths of the rivers Elbe and Weser is, at positive storm surges, effected by strong currents along the east Frisian coast. At heavy storms of 10 Beaufort, for example, they reach $90 \mathrm{~cm} / \mathrm{sec}$ at $4 \mathrm{~m}$ below the surface off Borkumriff, and $75 \mathrm{~cm} / \mathrm{sec}$ at $6 \mathrm{~m}$ below chart datum off Norderney. Even at $2 \mathrm{~m}$ above the sea floor a current of $40 \mathrm{~cm} / \mathrm{sec}$ was measured under these circumstances ar Borkumriff. The direction of these drift currents is rather constant even at considerably varying wind directions. Off the coast of Schleswig-Holstein the situation is far more complicated. At first, westerly winds transport the water towards the coast. But in the same manner as a large "Windstau" forms off the Elbe and Weser mouths, a strong west to north-west current develops at the Außeneider which removes the stemmed water, sometimes directly against the wind. This gradient current reaches $45 \mathrm{~cm} / \mathrm{sec}$ at heavy storms. Summarizing our findings, it can be said that water masses transported towards the east Frisian islands are removed from the coast of Schleswig-Holstein. At the Außeneider in particular in the range of the north Frisian islands, all current directions of the wind rose occur, depending on "Stau" and wind. The velocity rate lies at $25 \mathrm{~cm} / \mathrm{sec}$ during heavy storm. From November 17 to 21,1965 , strong easterly winds were prevailing over the German Bight and produced "negative storm surges" (with negative "Stau"). The current measurements recorded during this time indicate a drift current of about $30 \mathrm{~cm} / \mathrm{sec}$ in a westerly direction along the east Frisian islands, and a southerly gradient current of about the same rate in the area between P 8 and Außeneider. Fourier-integral-analyses of the current measurements revealed considerable long-wave oscillations in the part of the spectrum beyond the $\mathrm{M}_{2}$, if heavy storms have prevailed during the recording interval.
\end{abstract}

\section{EINLEITUNG}

Das Deutsche Hydrographische Institut hat während des Winters 1965/66 ein Arbeitsprogramm zur Messung der Strömungen in der Deutschen Bucht bei Sturm- 
fluten durchgeführt. Es ist von der Deutschen Forschungsgemeinschaft nachhaltig finanziell gefördert worden. Die Bewältigung der monatelangen umfangreichen Meßarbeit an vielen Beobachtungsstationen ist möglich geworden durch die ständige Unterstützung dieser Untersuchungen seitens der Wasser- und Schiffahrtsämter der deutschen Nordseeküste und deren Tonnenlegern mit ihren Kapitänen und Besatzungen. Dafür sei verbindlichst gedankt.

\section{ERGEBNISSE}

Die geographischen Positionen, an denen gemessen worden ist, zeigt Abbildung 1. An jedem der angegebenen Plätze sind durchweg zwei Geräte ausgelegt worden, eines $6 \mathrm{~m}$ unter Karten-Null und eines etwa $2 \mathrm{~m}$ über dem Boden. Darüber hinaus ist beim Vortrapp-Tief ein Strommesser $2 \mathrm{~m}$ unter der Wasseroberfläche ausgelegt worden, der leider während der Sturmfluten Ende Oktober bis Anfang November 1965 ausgefallen ist. Ferner sind die Vertikal-Log-Messungen des Feuerschiffes „Borkumriff“ für die Auswertungen herangezogen worden. Sie wurden $4 \mathrm{~m}$ unter der Wasseroberfläche gewonnen. In Abbildung 1 bedeutet $S$ Schaufelrad-Strommesser, $P$ Propellergerät (Tiefen- oder Flachseestrommesser). Die Zahl der Strompfeile in den Abbildungen stimmt mit der Zahl der Geräte durchweg nicht überein, weil Schäden an den Instrumenten oder auch Verluste auftraten. Außerdem sind wegen notwendiger Wartungsarbeiten nicht immer alle Geräte im Einsatz gewesen. Insgesamt sind während des Winters 1965/1966 im Rahmen dieses Meßprogramms 71 Auslegungen gemacht worden. Dabei sind 4 Geräte (2 Schaufelräder und 2 Tiefenstrommesser) verlorengegangen.

Zum Vergleich mit den Karten, die die Strömungsfelder bei Sturmfluten zeigen, gibt Abbildung 1 einen Uberblick über die Restströme (25stündige Mittel aus den Stromregistrierungen) bei ruhigem Wetter. Man hat also durchweg einen Reststrom von nur wenigen Zentimetern pro Sekunde. Lediglich vor der Elbe- und Wesermündung treten merklich größere Werte auf. Richtung und Betrag des Reststromes stimmen in beiden Meßniveaus überein.

Die besten Beobachtungen unseres Arbeitsprogramms sind während der Zeit vom 29. Oktober bis 4. November 1965 gemacht worden. Damals haben im Abstand von etwa 2 Tagen (am 30. Oktober und 1. November 1965) zwei Sturmtiefs die Nordsee überquert und Windverhältnisse in der Deutschen Bucht verursacht, die in Tabelle 1 dargestellt sind. Tabelle 2 gibt die als Folge dieser Wetterverhältnisse aufgetretenen Windstauwerte an.

Abbildung 2 bezieht sich auf den Beginn dieser Sturmflutperiode; 29. Oktober 1965, $3^{\text {h }} 38$ (ber. HW-Zeit von Cuxhaven). Man sieht, daß unter dem Einfluß des Windes entlang der ostfriesischen Küste ein östlicher Strom von etwa $6 \mathrm{~cm} / \mathrm{sec}$ entsteht. Vor der Elbe- und Wesermündung ist er bereits stärker und in der oberen Wasserschicht in höherem Maße auflandig als in der unteren. Der besonders starke Tiefenstrom bei Elbe 1 wird kinematische Ursachen haben. Vor der schleswig-holsteinischen Küste ist der Strom eindeutig auflandig. Der Windstau ist nur erst schwach ausgebildet.

Die Abbildung 3 kennzeichnet etwa 12 Stunden später die Situation beim ersten 


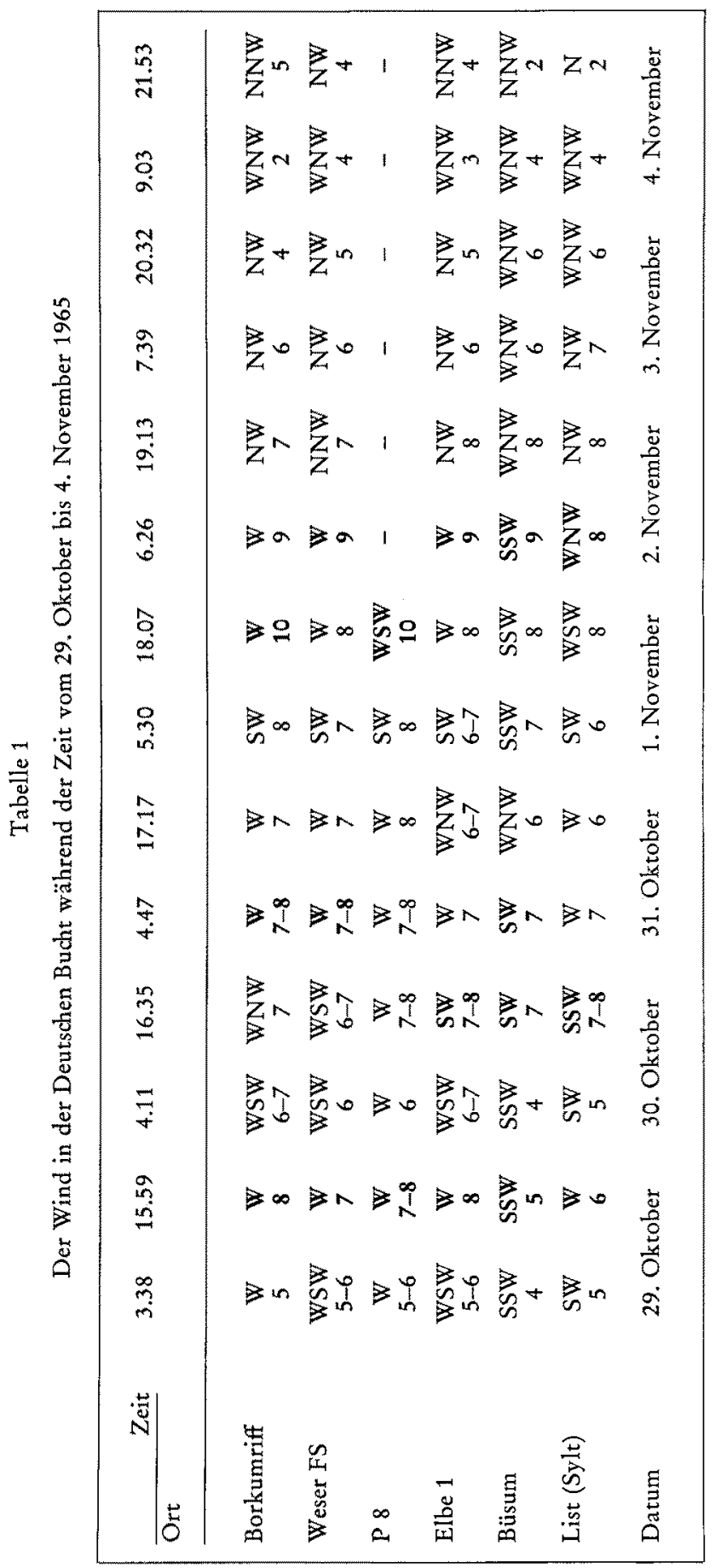


Strömungsmessungen bei Sturmfluten

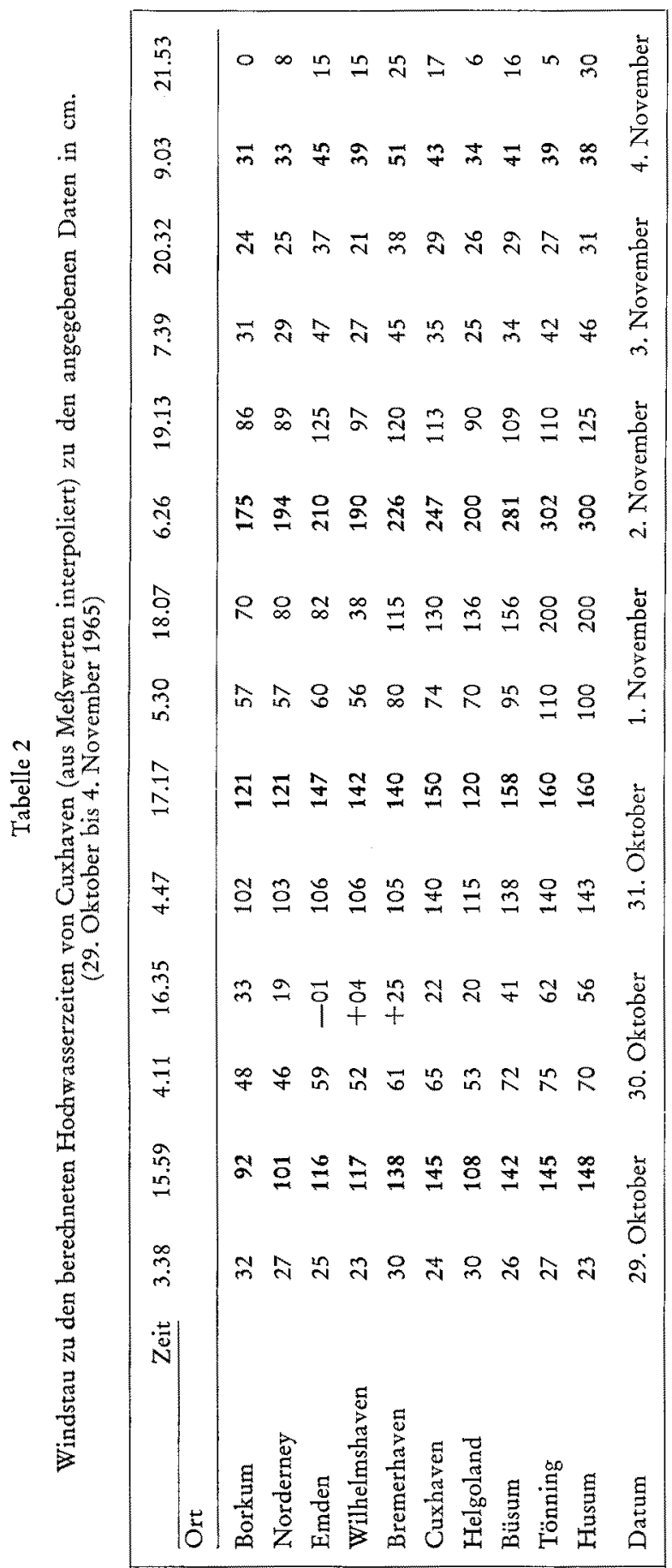




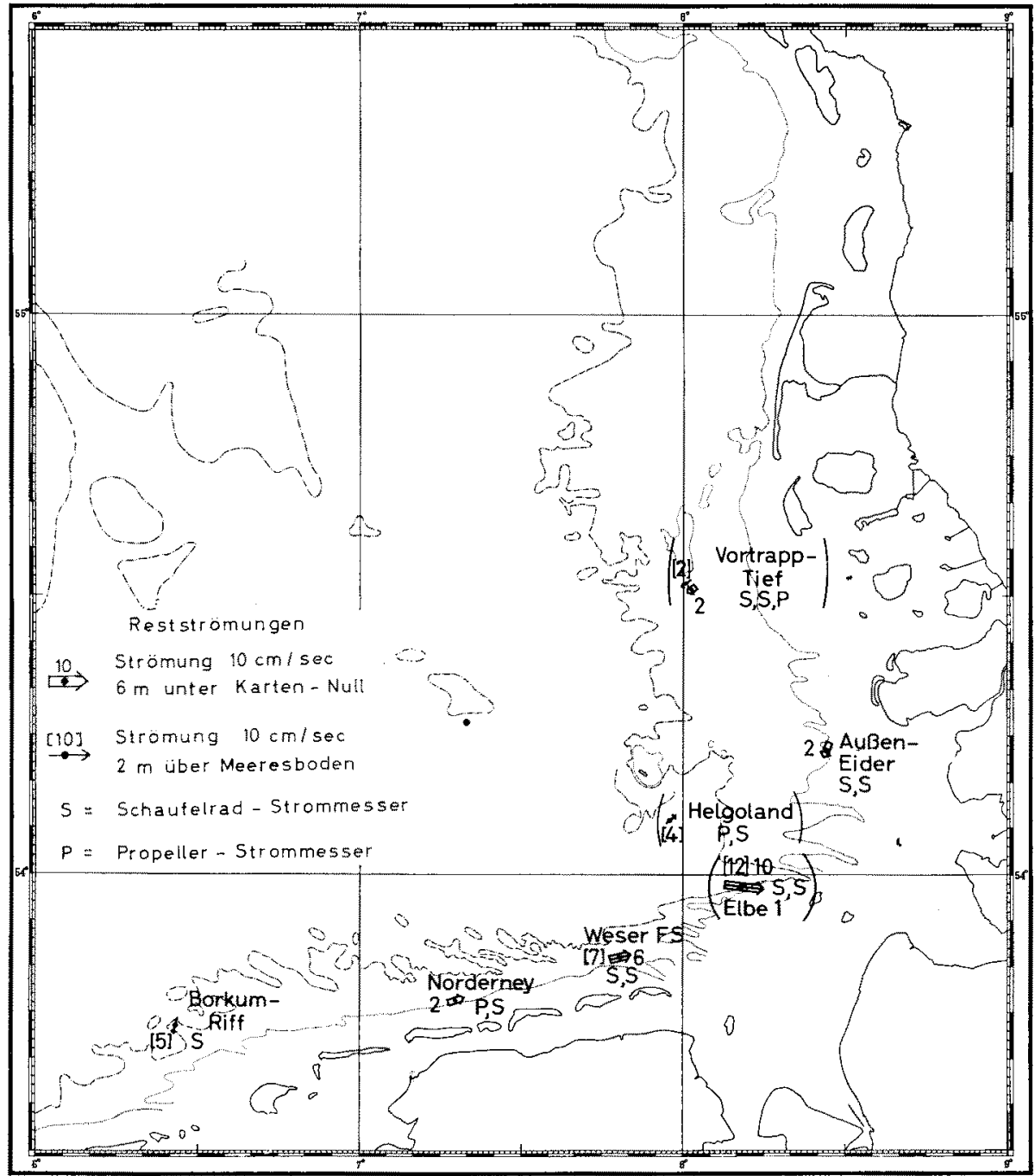

Abb. 1: Rest-Strömungen in verschiedenen Tiefen der Deutschen Bucht bei ruhigem Wetter. Die eingeklammerten Daten beziehen sich auf die Zeit vom 19. bis 21. Oktober 1965; die Werte ohne Klammern wurden vom 9. bis 12 . November 1965 gemessen. Einfache Pfeile zeigen die Strömungen an, die etwa $2 \mathrm{~m}$ über dem Meeresboden herrschen; dicke Pfeile geben die Messungen an, die $6 . \mathrm{m}$ unter Karten-Null vorgenommen wurden

Staumaximum, das mit dem ersten Windmaximum nahezu zusammenfällt. Der Stau ist gegenüber der Situation in Abbildung 2 stark angewachsen. Der östliche Strom zwischen Borkumriff und Norderney ist im wesentlichen erhalten geblieben. Bei Elbe 1 und Weser-Feuerschiff hat der Strom in der oberen Wasserschicht sich mehr vom Land abgedreht. Bei Außeneider ist ein Gefällstrom, dem Wind entgegen, entstanden, und 


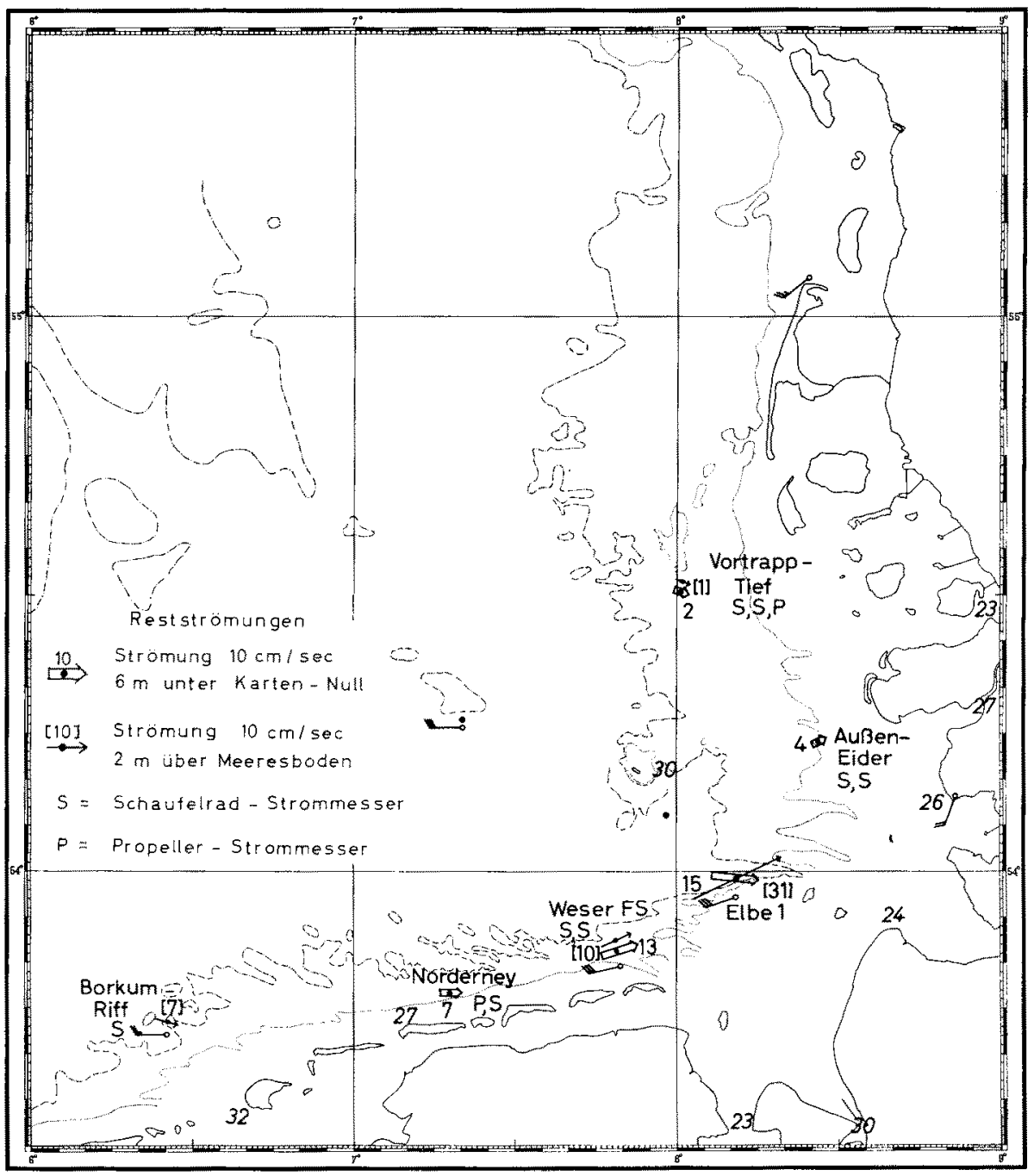

Abb. 2: Reststromfeld, Wind und Windstau in der Deutschen Bucht am 29. Oktober 1965 um 3.38 Uhr (errechnetes Hodhwasser in Cuxhaven). Zahlen in Kursiv-Schrif: Windstau

beim Vortrapp-Tief fließt zwar der Bodenstrom noch landwärts, aber der Oberflächenstrom dreht unter der Wirkung des durch den Stau bedingten Gefälles bereits seewärts.

Abbildung 4 zeigt, wiederum 12 Stunden später, das Ablaufen des Wassers vor der schleswig-holsteinischen Küste in einer Phase abnehmenden Windes und Staues. Auch beim Weser-Feuerschiff hat sich derStrom in der oberen Schicht seewärts gewandt, während er am Boden noch auflandig fließt. Bei Borkumriff dreht sich der Strom bereits allmählich in die für ruhiges Wetter charakteristische Richtung.

Den Höhepunkt einer schweren Sturmflut zeigt Abbildung 5. Die Situation ist 


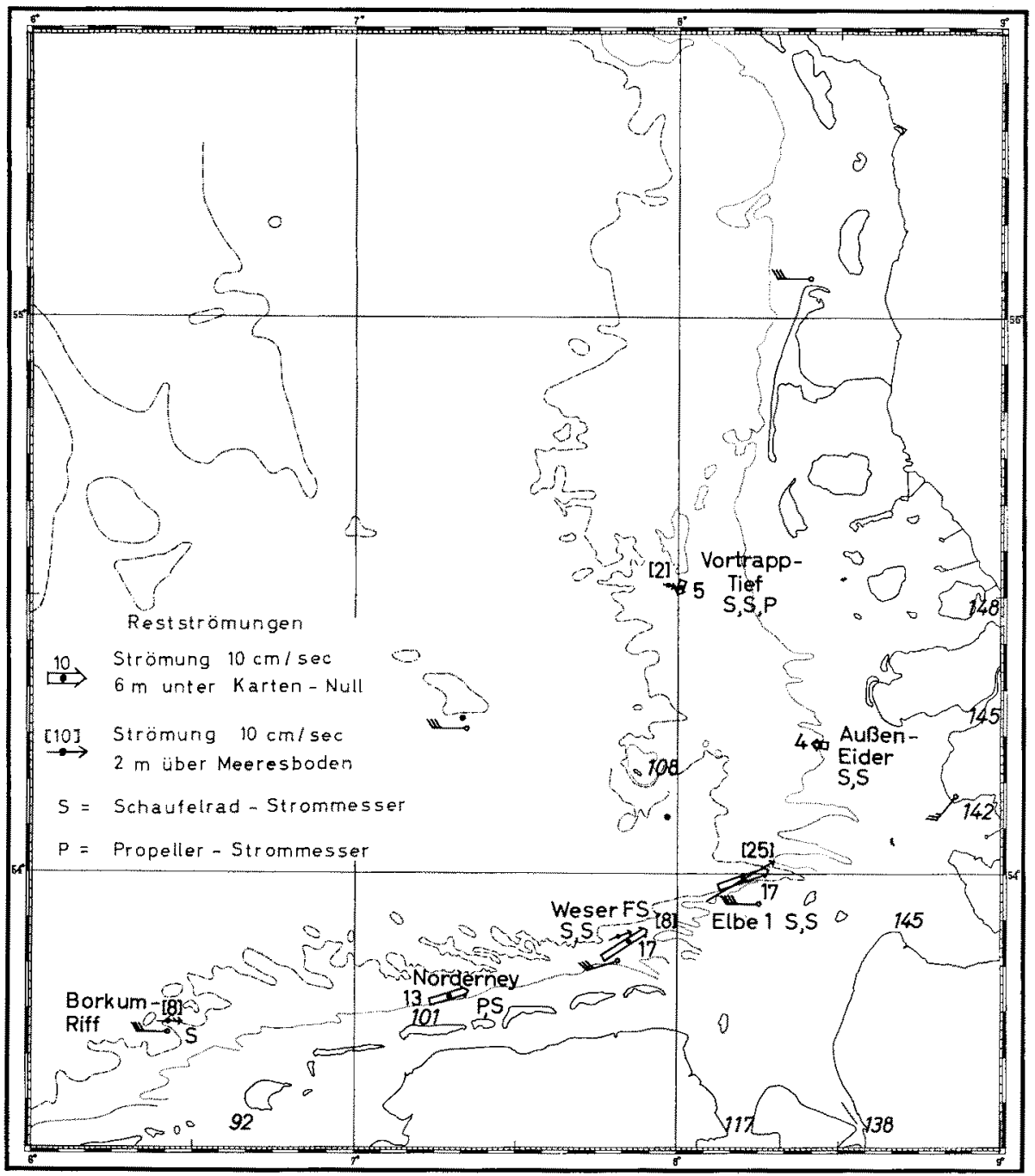

Abb. 3: Reststromfeld, Wind und Windstau in der Deutschen Bucht am 29. Oktober 1965 um 15.59 Uhr (errechnetes Hochwasser in Cuxhaven). Wind und Stau Maximum

gekennzeichnet durch ein Staumaximum, Maxima des Windes bei Elbe 1, Büsum und List sowie Strommaxima bei Borkumriff, Norderney und Außeneider. Die Ströme erreichen bei Borkumriff $4 \mathrm{~m}$ unter der Wasseroberfläche $90 \mathrm{~cm} / \mathrm{sec}$, und selbst $2 \mathrm{~m}$ über dem Boden ist noch $43 \mathrm{~cm} / \mathrm{sec}$ Strom gemessen worden. Bei Norderney ist der Betrag des Stroms $70 \mathrm{~cm} / \mathrm{sec}$, bei der Außeneider $40 \mathrm{~cm} / \mathrm{sec}$. Die extrem starke Bedeutung solcher Ereignisse für den Sandtransport ist offenkundig.

Abbildung 6 zeigt die Phase beginnender Abnahme des Windes und fallenden Staus, 12 Stunden später als in Abbildung 5 dargestellt. Obgleich der Wind auf Nord- 


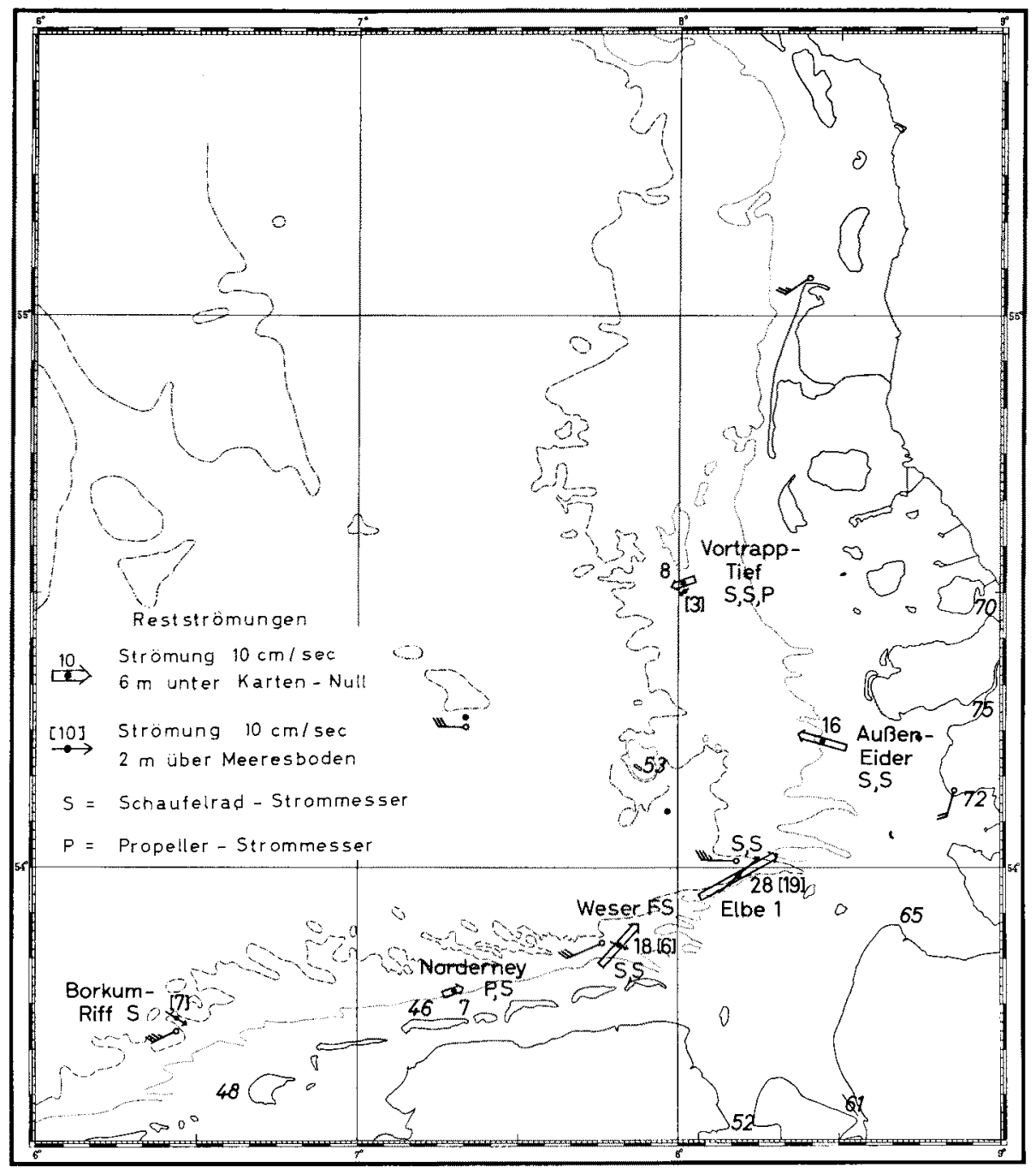

Abb. 4: Reststromfeld, Wind und Windstau in der Deutschen Bucht am 30. Oktober 1965 um 4.11 Uhr. Nachlassender Wind und Stau

west gedreht hat, sind die wesentlichen Züge des Stromfeldes erhalten geblieben. Bei Borkumriff und Helgoland fällt besonders das am Boden abfließende Wasser auf. Bei Elbe 1 ist der Bodenstrom nun gleichfalls stärker ablandig.

Im Zuge der weiteren Wind- und Stauabnahme zerfällt das Stromfeld. Beim Vortrapp-Tief entsteht dabei vorübergehend in der oberen Schicht ein östlicher Strom von $10 \mathrm{~cm} / \mathrm{sec}$, bei der Außeneider ein gleichgroßer von südlicher Richtung, während im Gebiet von Borkumriff bis Elbe 1 sich die für ruhige Wetterlagen typischen Restströme allmählich wieder einstellen. 


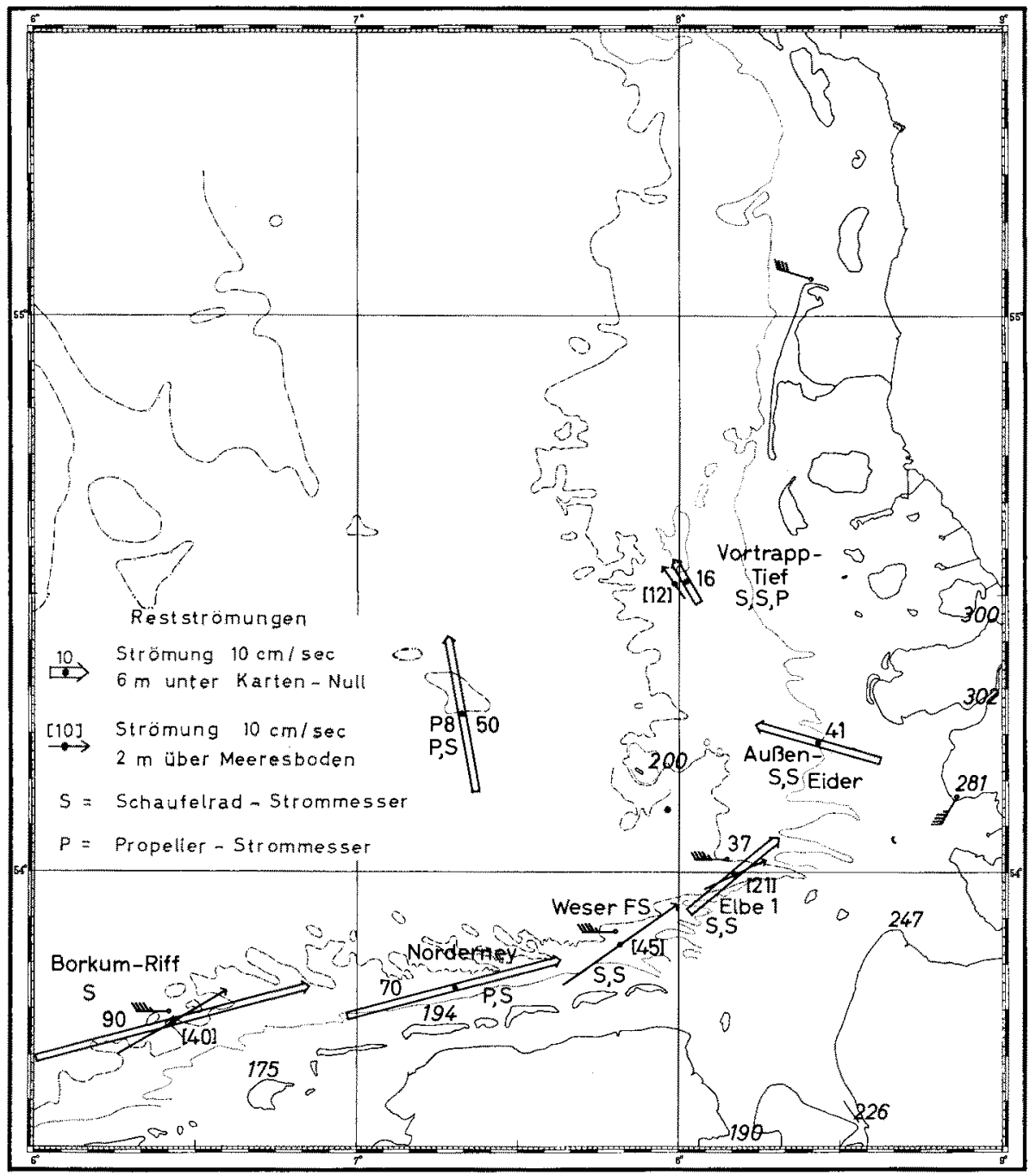

Abb. 5: Reststromfeld, Wind und Windstau in der Deutschen Bucht am 2. November 1965 um 6.26 Uhr. Maximalstau, Windmaximum bei Feuerschiff Elbe 1, Büsum und List. Strömungsmaxima bei Borkumriff, Norderney und Außeneider

In der Zeit vom 17. bis 21. November haben über der Deutschen Bucht starke Ostwinde geweht und „negative Sturmfluten“ veranlaßt. Die Strömungsmessungen aus dieser Zeit ergeben einen Driftstrom um $30 \mathrm{~cm} / \mathrm{sec}$ in westlicher Richtung entlang der ostfriesischen Inselkette und einen Gefällstrom etwa gleicher Größe im Gebiet P 8 - Außeneider von südlicher Richtung (Abb. 7). Der nach NNO gerichtete Stromvektor bei der Außeneider dreht im weiteren Verlauf der "negativen Sturmflut" südwärts. 


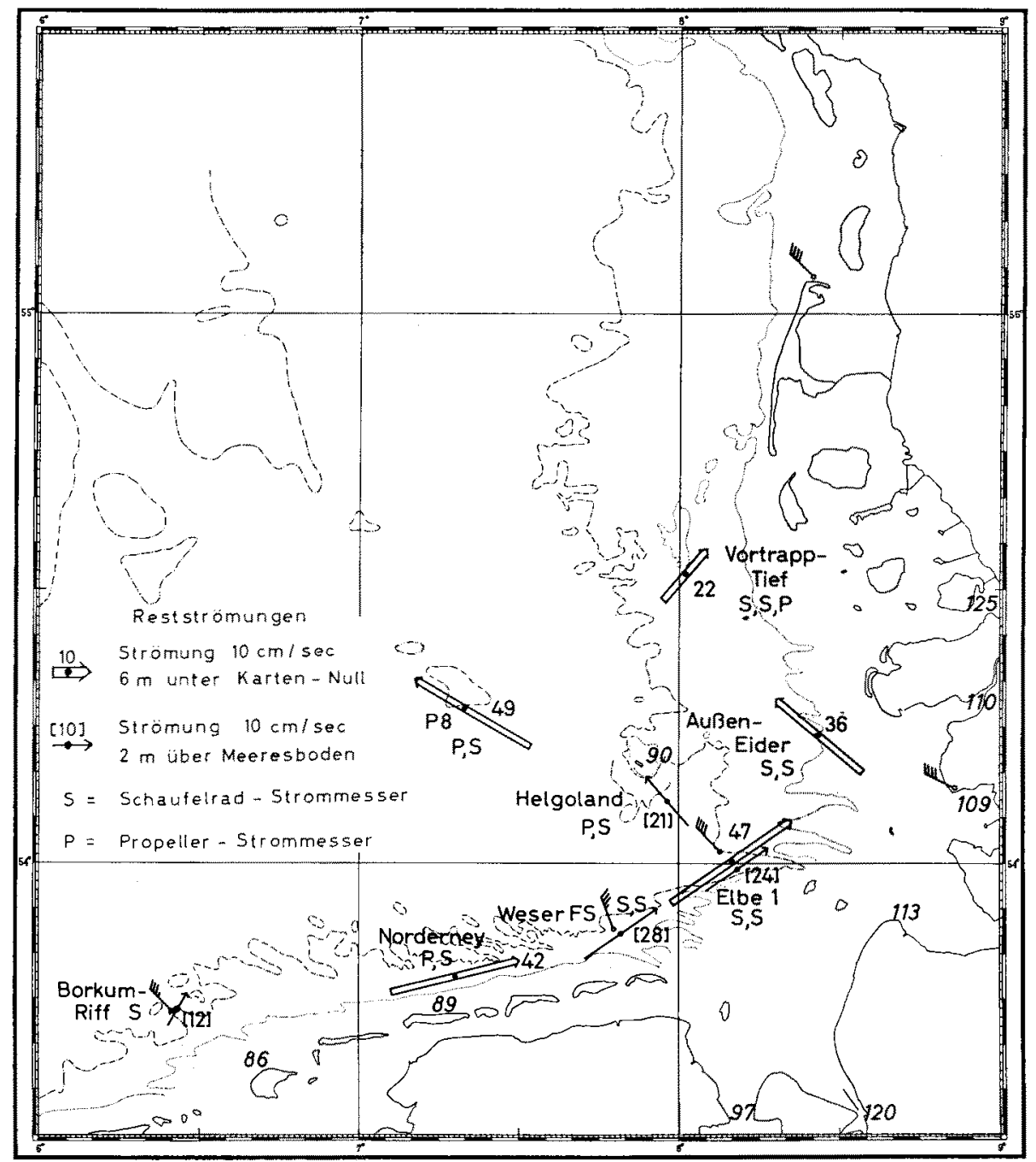

Abb. 6: Reststromfeld, Wind und Windstau am 2. November 1965, abnehmender Wind und Stau. (Bemerkenswert ist eine Strömung von ausfließendem Wasser südlich von Helgoland)

Zur Ermittlung des Frequenzspektrums der Strommessungen sind FourierIntegral-Analysen gerechnet worden für den Bereich der Schwingungszeiten von 3 bis 90 Stunden. Sie haben interessante Regelmäßigkeiten über das Verhalten des langwelligen Teiles des Spektrums jenseits der $\mathrm{M}_{2}$ ergeben. Dort treten nämlich intensive langwellige Schwingungen auf, sobald während der Registrierungszeit des Strommessers starke Stürme geherrscht haben (Abb. 8 bis 11). Besonders hervor treten die beiden Schwingungen mit Perioden um 35 und 60 Stunden. Sie kommen auch an anderen Beobachtungsstationen vor, wenn stürmische Wetterlagen gewesen sind, sonst 


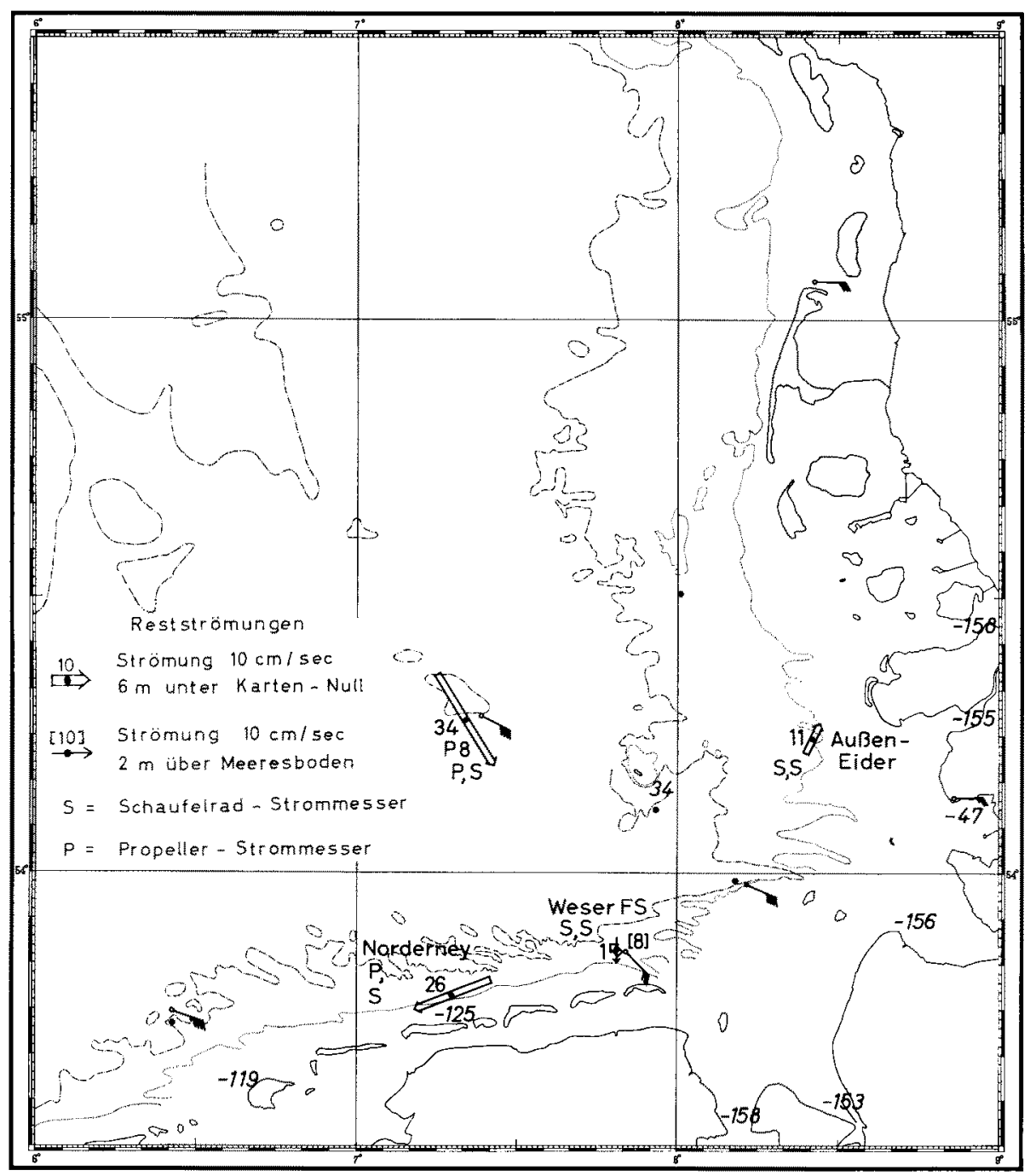

Abb. 7 : Eine "negative Sturmflut" in der Deutschen Bucht am 27. November 1965 um 19.53 Uhr infolge starken Ostwindes

nicht oder nur sehr schwach. Sie sind, wie es sein muß, an der Oberfläche stärker ausgebildet als am Boden. Die Tabelle 3 gibt eine Obersicht ïber das Auftreten langer Wellen in den Spektren von Stromregistrierungen. Die Amplituden der Stromschwingungen der intensivsten Wellen sind etwa fünfmal so groß wie die Fourieramplituden. $\mathrm{Zu}$ der 35-Stunden-Schwingung sind auch oft Oberschwingungen beobachtet worden. Setzt man in die Meriansche Formel $750 \mathrm{~km}$ und $60 \mathrm{~m}$ für die Länge und mittlere Tiefe der Nordsee ein, so erhält man gleichfalls eine Periode von 35 Stunden. Daher betrachten wir diesen Prozeß als nord-südliche Seiche der Nordsee. Der sehr großen 
Periode der 60-Stunden-Schwingung wegen ist es nicht möglich, sie gleichfalls als Seiche zu deuten. Wir nehmen daher an, daß sie als durch meteorologischen Einfluß erzwungene Schwingung aufgefaßt werden muß.

Tabelle 3

Das Auftreten langer Wellen in den Spektren von Stromregistrierungen. a und b bedeuten Messung am Boden bzw. in der oberen Schicht (vgl. Text). Die stärker umrandeten Felder zeigen an, daß Messungen mit Auswertungen vorliegen. Ein, zwei bzw. drei Kreuze bedeuten, daß schwache, mittelstarke bzw. starke Wellen beobachtet worden sind

\begin{tabular}{|c|c|c|c|c|}
\hline Ort & ruhig & stürmisch & ruhig & stürmisch \\
\hline \multicolumn{3}{|l|}{ AuBeneider } & & $\times x$ \\
\hline Vortrapp-Tief & $x$ & $x$ & & $x x$ \\
\hline \multicolumn{4}{|l|}{ P 8} & $x \times$ \\
\hline \multicolumn{5}{|l|}{ Helgoland } \\
\hline \multicolumn{2}{|l|}{ Elbe 1} & $x \times x$ & & $x \times x$ \\
\hline Weser & $x$ & $x$ & $x$ & $x$ \\
\hline \multicolumn{5}{|l|}{ Norderney } \\
\hline Borkumrif & & $x$ & & \\
\hline
\end{tabular}

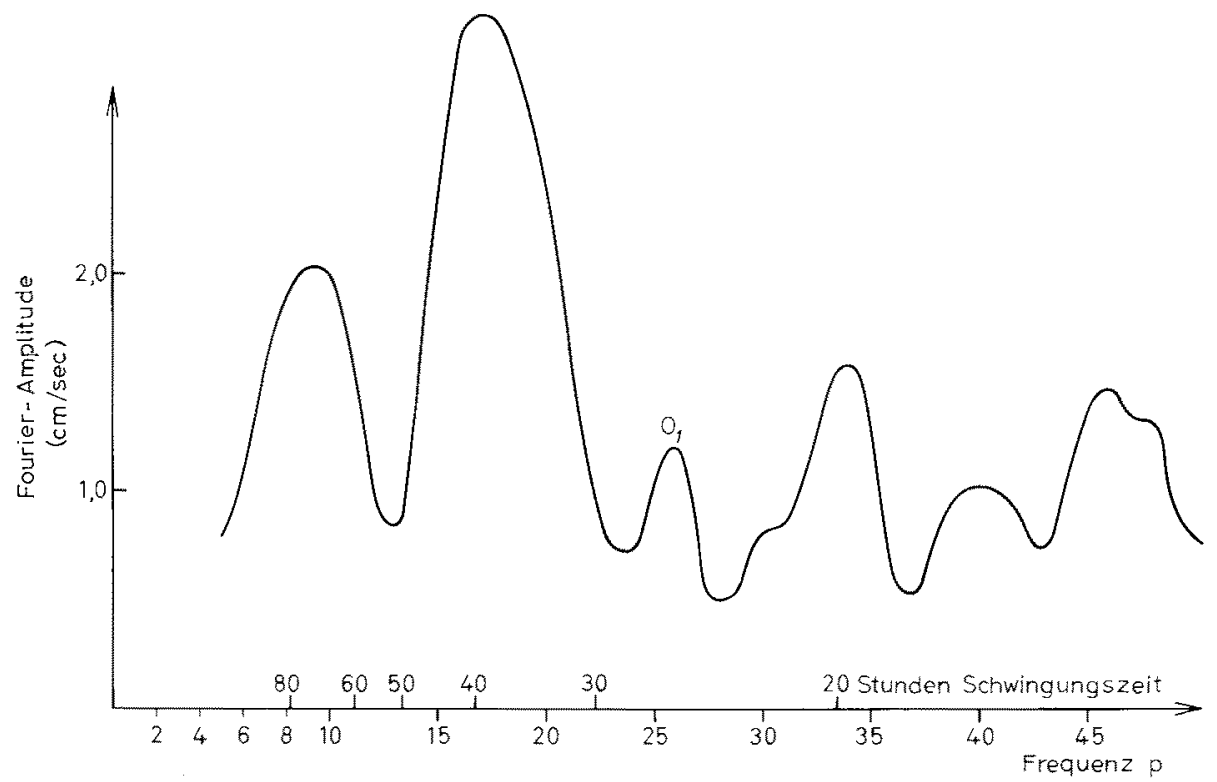

Abb. 8: Langwelliger Teil eines Spektrums von Strömungsmessungen bei der Außeneider; Nordkomponente; 19. Oktober bis 10. November 1965; Meßtiefe: $6 \mathrm{~m}$ unter KN; Station 4520; Ib 2 


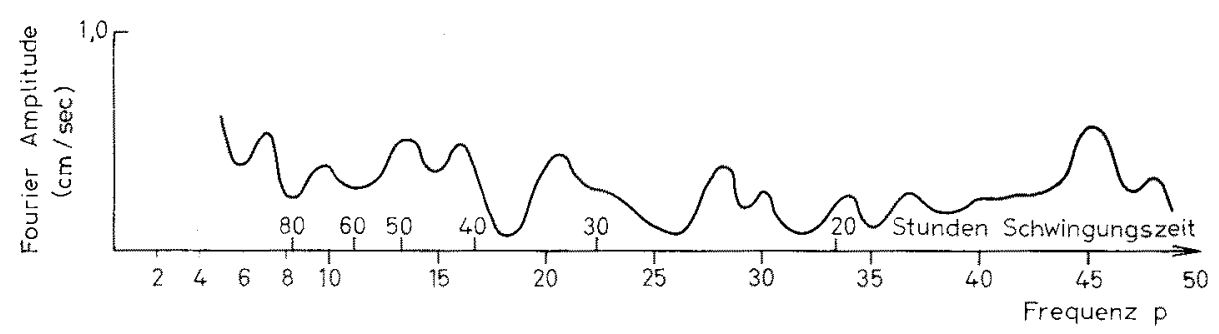

Abb. 9: Landwelliger Teil eines Spektrums von Strömungsmessungen bei der Außeneider; Nordkomponente; 27. September bis 18. Oktober 1965; Meßtiefe: $6 \mathrm{~m}$ unter KN; Station 4521; Ib 1

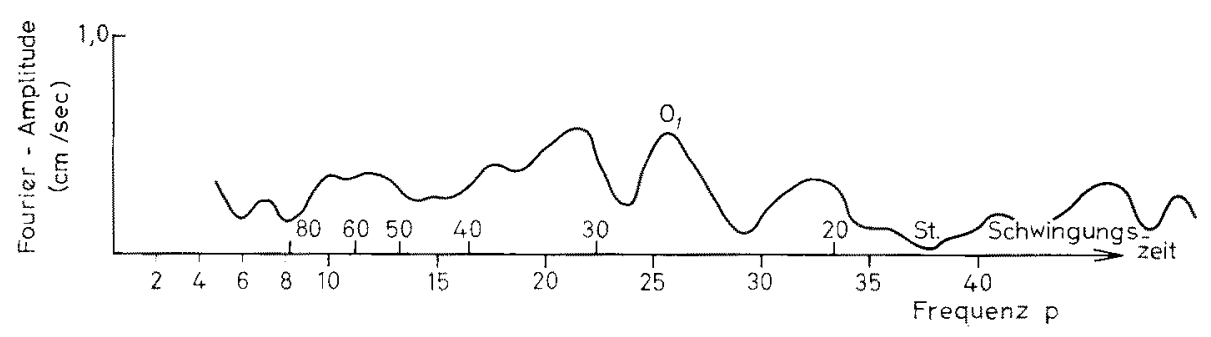

Abb. 10: Langwelliger Teil eines Spektrums von Strömungsmessungen beim Vortrapp-Tief; Nordkomponente; 19. Oktober bis 2. November 1965; Meßtiefe: $6 \mathrm{~m}$ unter KN; Station 4522; II 2

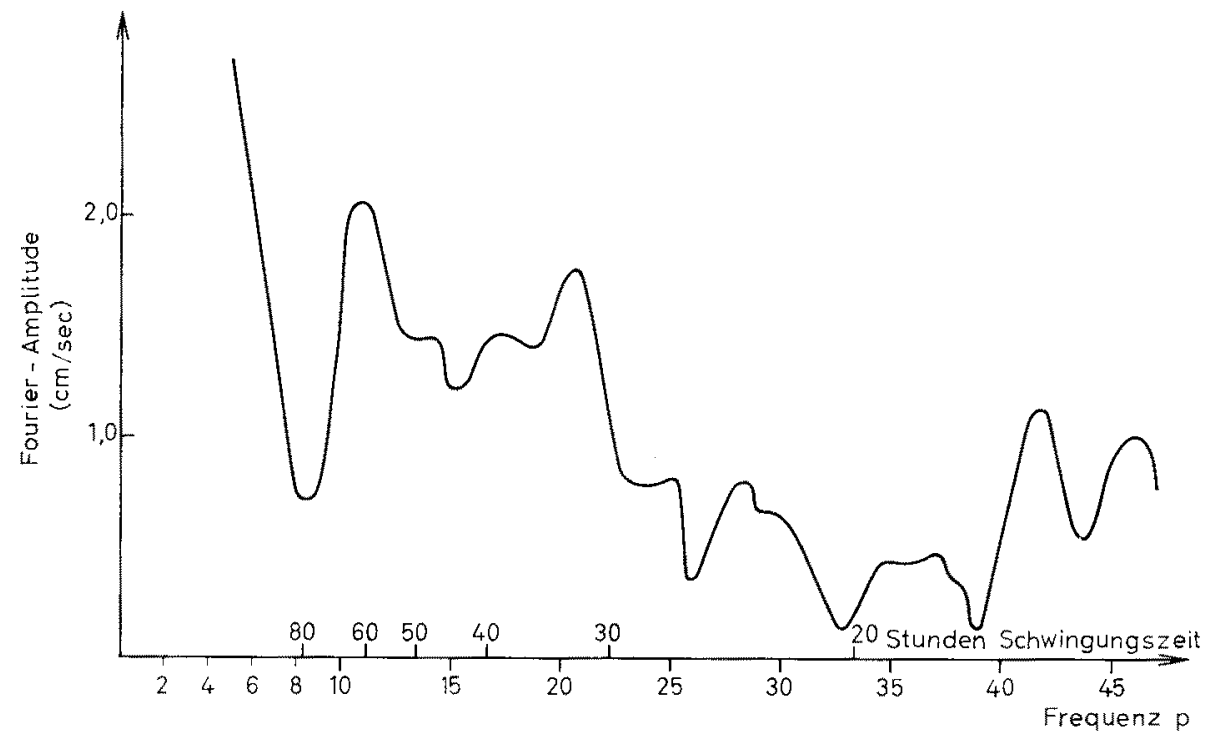

Abb. 11: Langwelliger Teil eines Spektrums von Strömungsmessungen beim Vortrapp-Tief; Nordkomponente; 19. Oktober bis 12. November 1965; Meßtiefe: $6 \mathrm{~m}$ unter KN; Station 4529; III 2 


\section{ZUSAMMENFASSUNG}

1. Während der Sturmfluten des Winters $1965 / 66$ sind an acht geographischen Positionen der Deutschen Bucht Strömungsmessungen in zwei, teilweise auch drei verschiedenen Tiefenlagen gemacht worden. Die Auswertung der Meßergebnisse hat Strömungsfelder positiver und negativer Sturmfluten (bei westlichen bzw. östlichen Winden) und Spektren der inneren Bewegungsvorgänge der Deutschen Bucht im Bereich der Schwingungszeiten von 3 bis 90 Stunden ergeben.

2. Eine typische Sturmflutperiode mit starken Winden aus SW bis NW trat vom 29. Oktober bis 4. November 1965 auf. Während dieser Zeit haben Strömungsmesser an folgenden Stellen ausgelegen: Vortrapp-Tief, Außeneider, P 8, Helgoland, Elbe I, Weser Feuerschiff, Norderney, Borkumriff. Ihre Meßergebnisse zeigen, daß der Antransport des Wassers in das Gebiet vor der Elbe- und Wesermündung bei positiven Sturmfluten durch starke Strömungen entlang der ostfriesischen Küste erfolgt. Sie erreichen beispielsweise bei schwerem Sturm der Windstärke 10 vor Borkumriff $4 \mathrm{~m}$ unter der Wasseroberfläche $90 \mathrm{~cm} / \mathrm{sec}$, vor Norderney in einer Wassertiefe $6 \mathrm{~m}$ unter K.N. $75 \mathrm{~cm} / \mathrm{sec}$. Selbst $2 \mathrm{~m}$ über dem Meeresboden ist unter diesen Umständen bei Borkumriff noch ein Strom von $40 \mathrm{~cm} / \mathrm{sec}$ gemessen worden. Die Richtung dieser Driftströmungen ist selbst bei wesentlichen Anderungen der Windrichtung recht stabil.

3. Vor der schleswig-holsteinischen. Westküste liegen die Verhältnisse wesentlich komplizierter. Zunächst transportiert der Westwind das Wasser auf die Küste zu. Jedoch entsteht in dem Maße, wie sich vor Elbe- und Wesermündung ein großer Windstau ausbildet, bei der Außeneider ein starker Strom in westlicher bis nordwestlicher Richtung, der das angestaute Wasser wieder abtransportiert, und zwar zeitweilig direkt gegen den Wind. Dieser Gefällstrom erreicht bei schwerem Sturm um $45 \mathrm{~cm} / \mathrm{sec}$.

4. Vor der schleswig-holsteinischen Küste erfolgt der Abtransport der Wassermassen, die entlang der ostfriesischen Inselkette herantransportiert worden sind.

5. Bei der Außeneider treten je nach dem Stau, besonders im Gebiet der Nordfriesischen Inseln, und dem Wind alle Stromrichtungen der Windrose auf. Der Betrag der Geschwindigkeit liegt bei schwerem Sturm um $25 \mathrm{~cm} / \mathrm{sec}$.

6. In der Zeit vom 17. bis 21. November 1965 haben über der Deutschen Bucht starke Ostwinde geweht und "negative Sturmfluten “ (mit negativem Stau) verursacht. Die Strömungsmessungen aus dieser Zeit ergeben einen Driftstrom um $30 \mathrm{~cm} / \mathrm{sec}$ in westlicher Richtung entlang der ostfriesischen Inselkette und einen Gefällstrom gleicher Größe im Gebiet P 8 - Außeneider von südlicher Richtung.

7. Die Fourier-Integral-Analyse der Strommessungen ergab, daß bei Stürmen im lang-welligen Teil des Spektrums jenseits der $M_{2}$ intensive zusätzliche Schwingungen mit Perioden um 35 und 65 Stunden auftreten. 